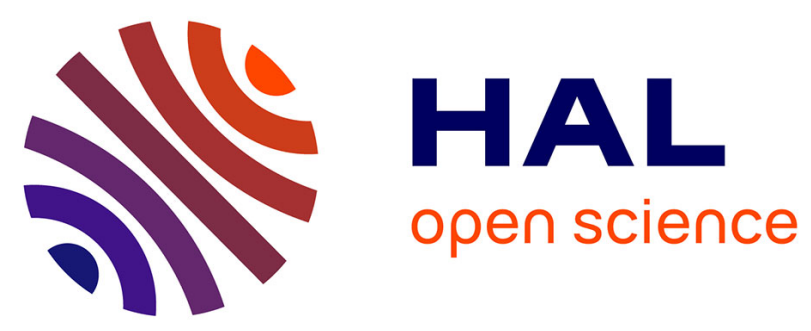

\title{
Stability analysis of asynchronous sampled-data systems with discrete-time constant input delay
}

Alexandre Seuret, Corentin Briat, Frédéric Gouaisbaut

\section{To cite this version:}

Alexandre Seuret, Corentin Briat, Frédéric Gouaisbaut. Stability analysis of asynchronous sampleddata systems with discrete-time constant input delay. 53th IEEE Conference on Decision and Control, Dec 2014, Los Angeles, CA, United States. pp. 4342-4347. hal-01065152

\section{HAL Id: hal-01065152 https://hal.science/hal-01065152}

Submitted on 18 Sep 2014

HAL is a multi-disciplinary open access archive for the deposit and dissemination of scientific research documents, whether they are published or not. The documents may come from teaching and research institutions in France or abroad, or from public or private research centers.
L'archive ouverte pluridisciplinaire HAL, est destinée au dépôt et à la diffusion de documents scientifiques de niveau recherche, publiés ou non, émanant des établissements d'enseignement et de recherche français ou étrangers, des laboratoires publics ou privés. 


\title{
Stability analysis of asynchronous sampled-data systems with discrete-time constant input delay
}

\author{
Alexandre Seuret, Corentin Briat and Frédéric Gouaisbaut
}

\begin{abstract}
Aperiodic sampled-data systems controlled by a delayed state-feedback controller arise naturally in networked control systems. We propose a novel way to analyze the stability of sampled-data systems with discrete-time constant input delay by, first, reformulating the system into an aperiodic discretetime system with constant delay and by, then, considering a mixture of a discrete-time Lyapunov-Krasovskii functional and a looped-functional in order to account for the presence of the delay and the sampling aperiodicity, respectively. Stability criteria, expressed as linear matrix inequalities, are derived for both certain and uncertain (time-varying) systems. An example is given for illustration.
\end{abstract}

\section{INTRODUCTION}

Sampled-data systems are a particular class of systems that have been extensively studied in the literature [1] as they arise, for instance, in digital control [2] and networked control systems [3], [4]. Several types of aperiodic sampling is not as well understood as the periodic case since the nonstationarity of the sampling process leads to additional difficulties in the analysis problem. Several approaches have been proposed in this regard. Discretetime systems approaches have been discussed in [5]-[8] where the sampling-period-dependent exponential terms in the stability conditions are embedded in a convex polytope, and are therefore considered as time-varying uncertainties. This approach leads to efficient and tractable conditions that can be easily used to design controllers. However, the approach is limited by the fact that only certain and timeinvariant systems can be considered. Another approach is based on the so-called "input-delay approach" and consists of reformulating the original sampled-data system into a timedelay system [9]-[12]. The system can then be analyzed using delay-techniques such as the Lyapunov-Krasovskii theorem. The main advantage of this approach lies in the possibility for considering uncertain, time-varying and even nonlinear systems. One limitation is the difficulty for designing controllers. Robust analysis techniques have also been applied in a successful way using small-gain results [13], IQCs [14]-[16] or well-posedness theory [17]. Approaches based on impulsive systems have also been considered using functionals [18] or clock-dependent Lyapunov functions [19].

A. Seuret and F. Gouaisbaut are with CNRS, LAAS-UPS, Univ Toulouse, 7 avenue du Colonel Roche, 31077 Toulouse, France \{aseuret, fgouaisb\}@laas.fr

C. Briat is with the Department of Biosystems Science and Engineering (D-BSSE), Swiss Federal Institute of Technology Zurich, Switzerland briatc@bsse. ethz.ch, corentin@briat. info, http://www.briat.info

This work is supported in part by the ANR project LimICoS contract number 12 BS03 00501 .
The latter approach has been shown to be able to both characterize the robust stability of periodic and aperiodic sampled-data systems in both the certain and (time-varying) uncertain cases. Interestingly, robust stabilization conditions for sampled-data systems can be easily formulated in a convex form using this approach. In this regard, this framework combines the efficiency of discrete-time and functional approaches. Finally, the looped-functional-based approach has been proposed in [20]-[22] to address the stability analysis problem of sampled-data and impulsive systems. This particular type of functional has the interesting property of not being necessarily required to be positive definite. Instead of that, we demand the fulfillment of a "looping condition", a certain boundary condition that can be made structurally satisfied while constructing the functional. In this regard, this class of functionals is therefore more general than usual Lyapunov(-Krasovsksii) functionals as the loopingcondition turns out to be weaker than the positive definiteness condition; see e.g. [20]-[22].

We propose to address the problem of stability analysis of (uncertain) aperiodic sampled-data systems with discretetime input delay. While the delayed sampled-data systems considered in [23], [24] are subject to a continuous-time delay (the delay is in seconds), the systems we are interested in here involve a discrete-time delay (the delay is a number of samples). A solution to this problem, based on state augmentation, has been proposed in [25]. The approach has been shown to yield accurate results but with a quite high computational complexity preventing then the application of the method when the delay is large. This is the reason why we will focus here on an alternative approach which is not based on state augmentation. Instead, the corresponding aperiodic discrete-time system with delay is directly considered and analyzed using an adequate blend of LyapunovKrasovskii and looped-functionals. The approach is applied to both certain and (time-varying) uncertain systems for which stability criteria are derived and formulated as LMIs.

Outline. Section III states preliminary technical results for dealing with LMIs and looped-functionals. The main stability results addressing both the nominal and robust stability of sampled-data systems with delay are presented in Section IV. An illustrative example is finally discussed in Section V.

Notations. Throughout the paper, $\mathbb{R}^{n}$ denotes the $n$ dimensional Euclidean space endowed with the vector 2norm $|\cdot|$. The space of $n \times m$ real matrices is denoted by $\mathbb{R}^{n \times m}$. For a matrix $P$, the notation $P \succ 0$ means that $P$ is symmetric and positive definite. The sets $\mathbb{S}^{n}$ and $\mathbb{S}_{+}^{n}$ are the set of $n$-dimensional symmetric and symmetric positive 
definite matrices, respectively.

\section{PROBLEM FORMULATION}

Let us consider here linear continuous-time systems of the form

$$
\begin{aligned}
& \dot{x}(t)=A x(t)+B u(t), t \geq 0, \\
& x(0)=x_{0}
\end{aligned}
$$

where $x \in \mathbb{R}^{n}$ and $u \in \mathbb{R}^{m}$ are the state of the system and the control input, respectively. Above, the matrices $A$ and $B$ may be uncertain and/or time-varying. This will be explicitly mentioned when this is the case. The control input $u$ obeys the following equation

$$
u(t)=K x\left(t_{k-h}\right), t \in\left[t_{k}, t_{k+1}\right), k \in \mathbb{N},
$$

where $K \in \mathbb{R}^{m \times n}$ is the controller gain and $h \in \mathbb{N}$ is the constant time-delay. The sequence $\left\{t_{k}\right\}_{k \in \mathbb{N}}, t_{0}=0$, is the sequence of sampling instants that we assume to obey the condition $T_{k}:=t_{k+1}-t_{k} \in\left[T_{\min }, T_{\max }\right]$ for all $k \in \mathbb{N}$ and for some $0<T_{\min } \leq T_{\max }<\infty$. Note that this condition implies that the sequence is increasing and admits no accumulation point, i.e. $t_{k} \rightarrow \infty$ as $k \rightarrow \infty$.

The closed-loop system obtained from the interconnection of (1) and (2) is given by

$$
\begin{aligned}
\dot{x}(t) & =A x(t)+B K x\left(t_{k-h}\right), t \in\left[t_{k}, t_{k+1}\right), k \in \mathbb{N}, \\
x\left(t_{-\theta}\right) & =\psi(\theta), \quad \theta=0, \ldots, h
\end{aligned}
$$

for some $\psi(\theta) \in \mathbb{R}^{n}$ with $\psi(0)=x_{0}$ and where $\left\{t_{-h}, \ldots, t_{-1}\right\}$ is a sequence of negative real numbers satisfying $t_{-h}<\ldots<t_{-1}<0$. Note that since only stability is considered, then $\psi(\theta)$ and $\left\{t_{-h}, \ldots, t_{-1}\right\}$ can be arbitrarily chosen. Alternatively, the above system can be represented by the time-delay discrete-time system

$$
\begin{aligned}
x\left(t_{k+1}\right) & =A_{d}\left(T_{k}\right) x\left(t_{k}\right)+B_{d}\left(T_{k}\right) x\left(t_{k-h}\right), k \in \mathbb{N}, \\
x\left(t_{-\theta}\right) & =\psi(\theta), \quad \theta=0, \ldots, h
\end{aligned}
$$

where $A_{d}\left(T_{k}\right)=e^{A T_{k}}$ and $B_{d}\left(T_{k}\right)=\int_{0}^{T_{k}} e^{A s} B K \mathrm{~d} s$.

Remark 1: It is important to stress that the system (3) dramatically differs from the system

$$
\begin{aligned}
& \dot{x}(t)=A x(t)+B K x\left(t_{k}-d\right), t \in\left[t_{k}, t_{k+1}\right) \\
& x(\theta)=\phi(\theta), \theta \in[-d, 0],
\end{aligned}
$$

defined for some delay $d>0$, that has been studied, for instance, in [26], [27], [23]. The delay here acts in a continuous way on the delayed term whereas in (3) the delays acts discretely. Note also that the above system is infinitedimensional while (3) is finite-dimensional. Both systems actually coincide whenever the sampling-period $T$ and the delay $h$ are constant and verify $d=h T$.

\section{PRELIMINARIES}

\section{A. A preliminary result}

The following result can be seen as a particular case of the reciprocally convex combination result derived in [28]:

Lemma 3.1 ( [29]): Let $R \in \mathbb{S}_{+}^{n}$ and $\alpha \in(0,1)$. Then, we have that

$$
\left[\begin{array}{cc}
R / \alpha & 0 \\
* & R /(1-\alpha)
\end{array}\right] \succeq\left[\begin{array}{c}
I \\
-I
\end{array}\right]^{\top} R\left[\begin{array}{c}
I \\
-I
\end{array}\right]
$$

Proof: We present here an alternative proof to the one in [29]. First note that we obviously have

$$
(1 / \alpha-1)(R-R) \succeq 0
$$

and, from the non-strict Schur complement [30] and the facts that $\alpha \in(0,1)$ and $R \succ 0$, we then obtain the equivalent condition

$$
\left[\begin{array}{cc}
(1 / \alpha-1) R & R \\
R & (1 /(1-\alpha)-1) R
\end{array}\right] \succeq 0
$$

where we have used the identity $(1 / \alpha-1)^{-1}=1 /(1-\alpha)-1$. This concludes the proof.

Note that the above bound is computationally less complex than the original one derived in [28] since no additional variable is introduced here. Regarding accuracy, it has been emphasized through several examples in [29] that the inequality from [28] and the one provided in Lemma 3.1 yield equivalent results for fast-varying delays systems.

\section{B. Lifting of sampled-data systems trajectories}

The looped-functional-based approach relies on the characterization of the trajectories of system (3) in a lifted domain [31], [21]. We therefore view the entire state-trajectory as a sequence of functions $\left\{x\left(t_{k}+\tau\right), \tau \in\left(0, T_{k}\right]\right\}_{k \in \mathbb{N}}$ with elements having a unique continuous extension to $\left[0, T_{k}\right]$ defined as

$$
\chi_{k}(\tau):=x\left(t_{k}+\tau\right) \quad \text { with } \quad \chi_{k}(0)=\lim _{s \downarrow t_{k}} x(s) .
$$

Finally, we define $\mathbb{K}$ as

$$
\mathbb{K}:=\underset{T \in\left[T_{\min }, T_{\max }\right]}{\bigcup \mathcal{C}\left([0, T] \rightarrow \mathbb{R}^{n}\right) .}
$$

Using this notation, the system (3) rewrites as

$$
\dot{\chi}_{k}(\tau)=A \chi_{k}(\tau)+B K \chi_{k-h}(0), \tau \in\left[0, T_{k}\right), k \in \mathbb{N} .
$$

In what follows, we also consider the notation

$$
\chi_{k}^{h}(\theta)=\chi_{k+\theta}(0)=x\left(t_{k+\theta}\right)
$$

where $\theta \in\{-h,-h+1, \ldots, 0\}$. We also denote by $\chi_{k}^{h}$ the vector $\operatorname{col}\left(\chi_{k}^{h}(0), \chi_{k}^{h}(-1), \ldots, \chi_{k}^{h}(-h)\right)$.

\section{Definition and results about looped-functionals}

Let us first define looped-functionals

Definition 3.2 ([21]): Let $0<T_{\min } \leq T_{\max }<\infty$. A functional

$$
f:\left[0, T_{\max }\right] \times \mathbb{K} \times\left[T_{\min }, T_{\max }\right] \rightarrow \mathbb{R},
$$

is said to be a looped-functional if

1) the equality

$$
f(0, z, T)=f(T, z, T)
$$

holds for all functions $z \in \mathbb{K}$ and all $T \in\left[T_{\min }, T_{\max }\right]$ and

2) it is differentiable with respect to the first variable with the standard definition of the derivative.

The set of all such functionals is denoted by $\mathfrak{L F}\left(\left[T_{\min }\right.\right.$, $\left.\left.T_{\max }\right]\right)$. 
The idea for proving stability of (3) is to look now for a positive definite quadratic form $V$ such that the sequence $\left\{V\left(\chi_{k}(0), \chi_{k}^{h}\right)\right\}_{k \in \mathbb{N}}$ is monotonically decreasing. This is formalized below through a functional existence result:

Theorem 3.3: Let $0<T_{\min } \leq T_{\max }<\infty, h \in \mathbb{N}$ and $V: \mathbb{R}^{n} \times \mathbb{R}^{n(h+1)} \rightarrow \mathbb{R}_{+}$be a quadratic form verifying

$$
\mu_{1}\left|\varphi_{2}\right|^{2} \leq V\left(\varphi_{1}, \varphi_{2}\right) \leq \mu_{2}\left|\varphi_{2}\right|^{2}
$$

for some scalars $0<\mu_{1} \leq \mu_{2}$ and for any $\left(\varphi_{1}, \varphi_{2}\right) \in$ $\mathbb{R}^{n} \times \mathbb{R}^{n(h+1)}$ where $\varphi_{2}=\left[\varphi_{1}^{\top} *\right]^{\top}$. Assume further that one of the following equivalent statements hold:

1) The sequence $\left\{V\left(\chi_{k}(0), \chi_{k}^{h}\right)\right\}_{k \in \mathbb{N}}$ is decreasing.

2) There exists a looped-functional $\mathcal{V} \in$ $\mathfrak{L F}\left(\left[T_{\min }, T_{\text {max }}\right]\right)$ such that the functional $\mathcal{W}_{k}$ defined as

$$
\begin{aligned}
\mathcal{W}_{k}\left(\tau, \chi_{k}, \chi_{k}^{h}\right):= & \frac{\tau}{T_{k}} \Lambda_{k}+V\left(\chi_{k}(\tau), \chi_{k}^{h}\right) \\
& +\mathcal{V}\left(\tau, \chi_{k}, T_{k}\right)
\end{aligned}
$$

with $\Lambda_{k}=V\left(\chi_{k+1}(0), \chi_{k+1}^{h}\right)-V\left(\chi_{k}\left(T_{k}\right), \chi_{k}^{h}\right)$ is decreasing along the trajectories of the system (7), i.e. the derivative

$$
\begin{aligned}
\frac{d}{d \tau} \mathcal{W}_{k}\left(\tau, \chi_{k}, \chi_{k}^{h}\right):= & \frac{1}{T_{k}} \Lambda_{k}+\frac{d}{d \tau} \mathcal{V}\left(\tau, \chi_{k}, T_{k}\right) \\
& +\frac{d}{d \tau} V\left(\chi_{k}(\tau), \chi_{k}^{h}\right)
\end{aligned}
$$

is negative definite for all $\tau \in\left[0, T_{k}\right], T_{k} \in$ $\left[T_{\min }, T_{\max }\right], k \in \mathbb{N}$.

Then, the system (3) with delay $h$ is asymptotically stable for any sequence $\left\{t_{k}\right\}_{k \in \mathbb{N}}$ satisfying $t_{k+1}-t_{k} \in$ $\left[T_{\min }, T_{\max }\right], k \in \mathbb{N}$.

Proof: The proof is similar to the one derived in [20], [21] and is thus omitted.

\section{STABility ANALYSis}

\section{A. Nominal stability analysis}

This section provides a stability result for aperiodic sampled-data systems with a constant delay $h$. We have the following result:

Theorem 4.1: The sampled-data system (7) with the delay $h$ is asymptotically stable for all $T_{k}:=t_{k+1}-t_{k} \in$ $\left[T_{\text {min }}, T_{\text {max }}\right], k \in \mathbb{N}$, if there exist matrices $R, Q, Z \in \mathbb{S}_{+}^{n}$, $P, X \in \mathbb{S}^{2 n}, S_{1}, S_{2} \in \mathbb{S}^{n}, U_{1}, U_{2} \in \mathbb{R}^{n \times n}$ such that the LMIs

$$
\begin{aligned}
\Phi_{0} & :=\left[\begin{array}{l}
I \\
I
\end{array}\right] P\left[\begin{array}{l}
I \\
I
\end{array}\right] \succ 0, \\
\Phi_{1}(\theta) & :=\left[\begin{array}{cc}
F_{0}(\theta)+\theta F_{1} & \theta M_{0}^{\top} Z \\
\star & -Z
\end{array}\right] \prec 0, \\
\Phi_{2}(\theta) & :=\left[\begin{array}{cc}
F_{0}(\theta)+\theta F_{2} & \theta M_{0}^{\top} Z \\
\star & -Z
\end{array}\right] \prec 0
\end{aligned}
$$

hold for all $\theta \in\left\{T_{\min }, T_{\max }\right\}$ where

$$
\begin{aligned}
F_{0}(\theta)= & F_{00}+\theta \operatorname{Sym}\left[\left[\begin{array}{c}
M_{1} \\
M_{3}
\end{array}\right]^{\top} P\left[\begin{array}{c}
M_{0} \\
0
\end{array}\right]\right] \\
& +F_{01}-F_{02}, \\
F_{00}= & M_{\Delta}^{\top} S_{1} M_{\Delta}+\operatorname{Sym}\left[\left(M_{\Delta}^{\top} U_{1}-M_{\delta}^{\top} U_{2}\right) M_{T}\right] \\
& -M_{\delta}^{\top} S_{2} M_{\delta} \\
F_{01}= & M_{2}^{\top} \Phi_{0} M_{2}-M_{T}^{\top} P M_{T}+M_{3}^{\top} Q M_{3} \\
& -M_{4}^{\top} Q M_{4}+h^{2} M_{\delta}^{\top} R M_{\delta}-M_{h}^{\top} R M_{h}, \\
F_{02}= & \left(M_{12}+M_{13}\right)^{\top} Z\left(M_{12}+M_{13}\right), \\
F_{1}= & \operatorname{Sym}\left[M_{0}^{\top}\left(S_{1} M_{\Delta}+U_{1} M_{T}\right)\right]-M_{T}^{\top} X M_{T}, \\
F_{2}= & \operatorname{Sym}\left[M_{0}^{\top}\left(S_{2} M_{\delta}+U_{2} M_{T}\right)\right]+M_{T}^{\top} X M_{T}
\end{aligned}
$$

with $M_{12}=M_{1}-M_{2}, M_{13}=M_{1}-M_{3}$ and

$$
\begin{aligned}
& M_{0}=\left[\begin{array}{llll}
A & 0 & 0 & B K
\end{array}\right], \quad M_{1}=\left[\begin{array}{llll}
I & 0 & 0 & 0
\end{array}\right] \text {, } \\
& M_{2}=\left[\begin{array}{llll}
0 & I & 0 & 0
\end{array}\right], \quad M_{3}=\left[\begin{array}{llll}
0 & 0 & I & 0
\end{array}\right], \\
& M_{4}=\left[\begin{array}{llll}
0 & 0 & 0 & I
\end{array}\right], \quad M_{\Delta}=\left[\begin{array}{llll}
I & -I & 0 & 0
\end{array}\right], \\
& M_{\delta}=\left[\begin{array}{llll}
0 & I & -I & 0
\end{array}\right], \quad M_{h}=\left[\begin{array}{llll}
0 & 0 & I & -I
\end{array}\right], \\
& M_{T}=\left[\begin{array}{ll}
M_{2}^{\top} & M_{3}^{\top}
\end{array}\right]^{\top} \text {. }
\end{aligned}
$$

Proof: See Appendix A.

As already mentioned in [20], [21], looped-functionals do not need to be positive definite for all $\tau \in\left[0, T_{k}\right]$, but need instead to satisfy the looping condition (8). In this regard, the class of looped-functionals is larger than the class of Lyapunov functionals considered, for instance, in [18], [12], [10], since the looping-condition is a weaker condition than the condition of positive definiteness. Note also that looped-functionals can incorporate information that is usually not considered in Lyapunov functionals. Notably, the term $\chi_{k}\left(T_{k}\right)$ is never involved in any of the Lyapunov functionals considered in the literature.

The novelty with respect to previous works is twofold. Firstly, the obtained conditions do not involve any additional decision variables; introduced for instance in the process of deriving the stability conditions from the derivative of the functional. This has been made possible via the use of the bound stated in Lemma 3.1. Secondly, the proposed method allows for the merging of a discrete-time stability criterion (although stated in continuous-time through the use of looped-functionals) to account for aperiodic sampling, together with a discrete-time Lyapunov-Krasovskii functional for dealing with the delay, in a simultaneous way. Until now, this combination of functionals has never been proposed anywhere else in the literature.

\section{B. Robust stability analysis}

One of the main advantages of the proposed method based on looped-functionals relies on the possibility for extending the stability conditions to uncertain systems. To this aim, let us consider now that the matrices of the system (3) are uncertain, possibly time-varying, and satisfy:

$$
\left[\begin{array}{ll}
A(t) & B(t)
\end{array}\right]=\sum_{i=1}^{N} \lambda_{i}(t)\left[\begin{array}{ll}
A_{i} & B_{i}
\end{array}\right]
$$

where $N$ is a positive integer and the matrices $A_{i}$ and $B_{i}$, $i=1, \ldots, N$, are known and of appropriate dimension. The 
time-varying parameters $\lambda_{i}(t), i=1, \ldots, N$, evolve within the $N$-unit simplex, that is we have

$$
\sum_{i=1}^{N} \lambda_{i}(t)=1, \quad \lambda_{i}(t) \geq 0, \quad i=1, \ldots, N, \quad t \geq 0 .
$$

We then have the following result:

Corollary 4.2: The uncertain sampled-data system (7)(13) with delay $h \in \mathbb{N}$ is asymptotically stable for all $T_{k}:=$ $t_{k+1}-t_{k} \in\left[T_{\min }, T_{\max }\right], k \in \mathbb{N}$ if there exist matrices $R, Q, Z \in \mathbb{S}_{+}^{n}, P, X \in \mathbb{S}^{2 n}, S_{1}, S_{2} \in \mathbb{S}^{n}, U_{1}, U_{2} \in \mathbb{R}^{n \times n}$ such that the LMIs

$$
\begin{gathered}
{\left[\begin{array}{l}
I \\
I
\end{array}\right] P\left[\begin{array}{l}
I \\
I
\end{array}\right] \succ 0,} \\
{\left[\begin{array}{cc}
F_{0}^{i}(\theta)+\theta F_{1}^{i} & \theta M_{0}^{i \top} Z \\
\star & -Z
\end{array}\right] \prec 0,} \\
{\left[\begin{array}{cc}
F_{0}^{i}(\theta)+\theta F_{2}^{i} & \theta M_{0}^{i \top} Z \\
\star & -Z
\end{array}\right] \prec 0}
\end{gathered}
$$

hold for all $\theta \in\left\{T_{\min }, T_{\max }\right\}$ with

$$
\begin{aligned}
F_{0}^{i}(\theta)= & F_{00}+\theta \operatorname{Sym}\left[\left[\begin{array}{l}
M_{1} \\
M_{3}
\end{array}\right]^{\top} P\left[\begin{array}{c}
M_{0}^{i} \\
0
\end{array}\right]\right] \\
& +F_{01}-F_{02}, \\
F_{1}^{i}= & \operatorname{Sym}\left[M_{0}^{i \top}\left(S_{1} M_{\Delta}+U_{1} M_{T}\right)\right]-M_{T}^{\top} X M_{T}, \\
F_{2}^{i}= & \operatorname{Sym}\left[M_{0}^{i \top}\left(S_{2} M_{\delta}+U_{2} M_{T}\right)\right]+M_{T}^{\top} X M_{T},
\end{aligned}
$$

where $M_{0}^{i}=\left[\begin{array}{llll}A_{i} & 0 & 0 & B_{i} K\end{array}\right]$ and all the others $M$ 's matrices are defined in Theorem 4.1.

Proof: The proof follows from the fact that the LMI conditions of Theorem 4.1 are affine in the matrix $M_{0}$. Noting finally that

$$
\begin{aligned}
M_{0}(t) & =\left[\begin{array}{llll}
A(t) & 0 & 0 & B(t) K
\end{array}\right] \\
& =\sum_{i=1}^{N} \lambda_{i}(t)\left[\begin{array}{llll}
A_{i} & 0 & 0 & B_{i} K
\end{array}\right]
\end{aligned}
$$

then the result follows from a simple convexity argument.

\section{EXAMPLE} [32]:

Let us consider the sampled-data system (3) with matrices

$$
A_{0}=\left[\begin{array}{cc}
0 & 1 \\
0 & -0.1
\end{array}\right], B_{0}=\left[\begin{array}{c}
0 \\
-0.1
\end{array}\right] \text { and } K=[3.75,11.5]
$$

In the case of constant sampling-period $T$, we can determine, for any delay value $h$, the maximal sampling-period that still preserves the stability of the system (3)-(15) by checking at the location of the eigenvalues of the matrix governing the corresponding discrete-time system. These values are upperbounds to the maximal admissible $T_{\max }$ in the aperiodic-case since stability regions of aperiodic sampled-data systems are included in those of periodic sampled-data systems. We then use Theorem 4.1 to analyze the system (3)-(15) in both the periodic and aperiodic sampling cases. The results are shown in Table I and Table II, respectively.
We can see that, in the periodic case, i.e. Table I, the conditions stated in Theorem 4.1 are almost nonconservative when the delay $h$ is small, but that this conservatism increases as the delay $h$ increases. Note, however, that the proposed approach yields results that are competitive with previously obtained ones.

On the other hand, we can see, from Table II, that the method proposed in [25] is able to yield more accurate results than the proposed one in the aperiodic sampling case. This stems from the fact that a tighter bound than the one stated in Lemma 3.1 is used in [25] in order to derive stability criteria. Note, however, that the computational complexity of the current approach scales much better than the approach considered in [25]. The complexity of the conditions derived in this latter reference indeed increases exponentially with the delay $h$, making the method quite impractical for large values of the delay. In this regard, the proposed approach shows a good tradeoff between accuracy and complexity.

TABLE I

MAXIMAL STABILITY-PRESERVING PERIODIC SAMPLING PERIOD $\bar{T}=T_{\max }=T_{\min }$ FOR THE SAMPLED-DATA SYSTEM (3)-(15) FOR DIFFERENT VALUES OF THE DELAY $h$.

\begin{tabular}{|l||c|c|c|c|c|}
\hline$h$ & 0 & 1 & 2 & 5 & 10 \\
\hline Theoretical bounds & 1.729 & 0.763 & 0.463 & 0.216 & 0.112 \\
\hline \hline$[27]$ (with $\tau=h T)$ & 1.278 & 0.499 & 0.333 & 0.166 & 0.090 \\
$[33]$ (with $\tau=h T)$ & 1.638 & 0.573 & 0.371 & 0.179 & 0.096 \\
$[23]$ (with $\tau=h T)$ & 1.721 & 0.701 & 0.431 & 0.197 & 0.103 \\
[25] & 1.729 & 0.763 & 0.463 & $-* 1$ & $-* 1$ \\
\hline Theorem 4.1 & 1.728 & 0.761 & 0.448 & 0.199 & 0.103 \\
\hline
\end{tabular}

\section{TABLE II}

MAXIMAL STABILITY-PRESERVING $T_{\max }\left(\right.$ WITH $T_{\min }=10^{-2}$ ) FOR THE APERIODIC SAMPLED-DATA SYSTEM (3)-(15) FOR DIFFERENT VALUES OF THE DELAY $h$.

\begin{tabular}{|l||c|c|c|c|c|}
\hline$h$ & 0 & 1 & 2 & 5 & 10 \\
\hline$[25]$ & 1.729 & 0.763 & 0.463 & $-^{* 1}$ & $-^{* 1}$ \\
\hline Theorem 4.1 & 1.708 & 0.618 & 0.377 & 0.176 & 0.094 \\
\hline
\end{tabular}

\section{CONCLUSION}

A novel way for analyzing stability of periodic and aperiodic uncertain sampled-data systems with discrete-time delays has been presented. Instead of using a discretetime criterion that would prevent the generalization of the approach to uncertain systems with time-varying uncertainties, an alternative approach based on looped-functionals and Lyapunov-Krasovskii functionals has been preferred. The novelty of the method is twofold. First, the stability conditions are free of slack variables, hence numerically less complex than those of the literature, while still being competitive with them in terms of conservatism, at least, for the considered example. Second, the obtained stability conditions are derived from the combination of looped-functionals

\footnotetext{
${ }^{1}$ The entry "-_"” in the tables means that the conditions were not checked due to an unreasonable computational complexity.
} 
and Lyapunov-Krasovskii functionals, an approach that has never been explored before.

Future works will be devoted to the extension to the timevarying delay case and on the use of tighter inequalities in order to reduce the overall conservatism of the approach.

\section{APPENDIX}

\section{A. Proof of Theorem 4.1}

Let us consider the Lyapunov function for the discretetime system (4) given by

$$
\begin{aligned}
V\left(\chi_{k}(\tau), \chi_{k}^{h}\right)= & {\left[\begin{array}{c}
\chi_{k}(\tau) \\
\chi_{k}(0)
\end{array}\right]^{\top} P\left[\begin{array}{l}
\chi_{k}(\tau) \\
\chi_{k}(0)
\end{array}\right] } \\
& +\sum_{i=k-h}^{k-1} \chi_{i}^{\top}(0) Q \chi_{i}(0) \\
& +h \sum_{i=-h}^{-1} \sum_{j=k+i}^{k-1} \delta_{i}^{\top}(0) R \delta_{i}(0)
\end{aligned}
$$

where $\delta_{i}(0)=\chi_{i+1}(0)-\chi_{i}(0)$. Let us also define the functional $\mathcal{V}$ as

$$
\begin{aligned}
T_{k} \mathcal{V} & \left(\tau, \chi_{k}, T_{k}\right)=\tau\left(T_{k}-\tau\right)\left[\begin{array}{c}
\chi_{k}\left(T_{k}\right) \\
\chi_{k}(0)
\end{array}\right]^{\top} X\left[\begin{array}{c}
\chi_{k}\left(T_{k}\right) \\
\chi_{k}(0)
\end{array}\right] \\
& +\tau\left(\chi_{k}(\tau)-\chi_{k}\left(T_{k}\right)\right)^{\top} S_{1}\left(\chi_{k}(\tau)-\chi_{k}\left(T_{k}\right)\right) \\
& +\left(T_{k}-\tau\right)\left(\chi_{k}(\tau)-\chi_{k}(0)\right)^{\top} S_{2}\left(\chi_{k}(\tau)-\chi_{k}(0)\right) \\
& +2 \tau\left(\chi_{k}(\tau)-\chi_{k}\left(T_{k}\right)\right)^{\top} U_{1}\left[\begin{array}{c}
\chi_{k}\left(T_{k}\right) \\
\chi_{k}(0)
\end{array}\right] \\
& +2\left(T_{k}-\tau\right)\left(\chi_{k}(\tau)-\chi_{k}(0)\right)^{\top} U_{2}\left[\begin{array}{c}
\chi_{k}\left(T_{k}\right) \\
\chi_{k}(0)
\end{array}\right] \\
& +T_{k}^{2} \int_{0}^{\tau} \dot{\chi}_{k}(s)^{\top} Z \dot{\chi}_{k}(s) \mathrm{d} s \\
& -T_{k} \tau \int_{0}^{T_{k}} \dot{\chi}_{k}(s)^{\top} Z \dot{\chi}_{k}(s) \mathrm{d} s
\end{aligned}
$$

where $Z \in \mathbb{S}_{+}^{n}, S_{1}, S_{2} \in \mathbb{S}^{n}, X \in \mathbb{S}^{2 n}$ and $U_{1}, U_{2} \in \mathbb{R}^{n \times 2 n}$. Note that this functional verifies the equality

$$
\mathcal{V}\left(0, \chi_{k}, T_{k}\right)=\mathcal{V}\left(T_{k}, \chi_{k}, T_{k}\right)=0
$$

for all $T_{k} \in\left[T_{\min }, T_{\max }\right]$. Following Theorem 3.3, we consider

$$
\begin{aligned}
\dot{\mathcal{W}}_{k}\left(\tau, \chi_{k}, \chi_{k}^{h}\right)= & \frac{1}{T_{k}}\left(\Lambda_{k}+T_{k} \dot{V}\left(\chi_{k}(\tau), \chi_{k}^{h}\right)\right. \\
& \left.+T_{k} \dot{\mathcal{V}}\left(\tau, \chi_{k}, T_{k}\right)\right)
\end{aligned}
$$

where $\Lambda_{k}$ is defined in Theorem 3.3. The asymptotic stability of the system (7) is established if we can prove 1) the positive definiteness of $V\left(\chi_{k}(0), \chi_{k}^{h}\right)$ and 2) the negative definiteness of $\dot{\mathcal{W}}_{k}$. Note first that $V\left(\chi_{k}(0), \chi_{k}^{h}\right)$ is positive definite if $\Phi_{0}, Q$ and $R$ are also positive definite. This proves the first condition. Let us now focus on the second one and, to this aim, we define the augmented vector

$$
\xi_{k}(\tau):=\left[\begin{array}{c}
\chi_{k}(\tau) \\
\chi_{k}\left(T_{k}\right) \\
\chi_{k}(0) \\
\chi_{k-h}(0)
\end{array}\right] .
$$

We then have

$$
\begin{aligned}
\Lambda_{k}= & {\left[\begin{array}{c}
\chi_{k+1}(0) \\
\chi_{k+1}(0)
\end{array}\right]^{\top} P\left[\begin{array}{c}
\chi_{k+1}(0) \\
\chi_{k+1}(0)
\end{array}\right]+\chi_{k}^{\top}(0) Q \chi_{k}(0) } \\
- & {\left[\begin{array}{c}
\chi_{k}\left(T_{k}\right) \\
\chi_{k}(0)
\end{array}\right]^{\top} P\left[\begin{array}{c}
\chi_{k}\left(T_{k}\right) \\
\chi_{k}(0)
\end{array}\right]-\chi_{k-h}^{\top}(0) Q \chi_{k-h}(0) } \\
& +h \sum_{i=k-h+1}^{k} \delta_{i}^{\top}(0) R \delta_{i}(0)
\end{aligned}
$$

Since $\chi_{k+1}(0)=\chi_{k}\left(T_{k}\right)$, the previous expression can easily be reformulated in terms of the augmented vector $\xi_{k}(\tau)$. Applying then Jensen's inequality to the summation term yields

$$
\begin{aligned}
\Lambda_{k} \leq & \xi_{k}^{\top}(\tau)\left(M_{2}^{\top} \Phi_{0} M_{2}-\left[\begin{array}{c}
M_{2} \\
M_{3}
\end{array}\right]^{\top} P\left[\begin{array}{l}
M_{2} \\
M_{3}
\end{array}\right]\right. \\
& +M_{3}^{\top} Q M_{3}-M_{4}^{\top} Q M_{4} \\
& \left.+h^{2} M_{\delta}^{\top} R M_{\delta}-M_{h}^{\top} R M_{h}\right) \xi_{k}(\tau) \\
= & \xi_{k}^{\top}(\tau) F_{01} \xi_{k}(\tau) .
\end{aligned}
$$

Note that we also have

$$
\begin{aligned}
\dot{V}\left(\chi_{k}(\tau), \chi_{k}^{h}\right) & =2\left[\begin{array}{l}
\chi_{k}(\tau) \\
\chi_{k}(0)
\end{array}\right]^{\top} P\left[\begin{array}{c}
\dot{\chi}_{k}(\tau) \\
0
\end{array}\right] \\
& =2 \xi_{k}^{\top}(\tau)\left[\begin{array}{c}
M_{1} \\
M_{3}
\end{array}\right]^{\top} P\left[\begin{array}{c}
M_{0} \\
0
\end{array}\right] \xi_{k}(\tau),
\end{aligned}
$$

and, finally,

$$
\begin{aligned}
T_{k} \dot{\mathcal{V}} & \left.\tau, \chi_{k}, T_{k}\right)=\left(T_{k}-2 \tau\right)\left[\begin{array}{c}
\chi_{k}\left(T_{k}\right) \\
\chi_{k}(0)
\end{array}\right]^{\top} X\left[\begin{array}{c}
\chi_{k}\left(T_{k}\right) \\
\chi_{k}(0)
\end{array}\right] \\
& +\left(\chi_{k}(\tau)-\chi_{k}\left(T_{k}\right)\right)^{\top} S_{1}\left(\chi_{k}(\tau)-\chi_{k}\left(T_{k}\right)\right) \\
& -\left(\chi_{k}(\tau)-\chi_{k}(0)\right)^{\top} S_{2}\left(\chi_{k}(\tau)-\chi_{k}(0)\right) \\
& +2 \tau \dot{\chi}_{k}^{\top}(\tau) S_{1}\left(\chi_{k}(\tau)-\chi_{k}\left(T_{k}\right)\right) \\
& +2\left(T_{k}-\tau\right) \dot{\chi}_{k}^{\top}(\tau) S_{2}\left(\chi_{k}(\tau)-\chi_{k}(0)\right) \\
& +2\left(\chi_{k}(\tau)-\chi_{k}\left(T_{k}\right)\right)^{\top} U_{1}\left[\begin{array}{c}
\chi_{k}\left(T_{k}\right) \\
\chi_{k}(0)
\end{array}\right] \\
& -2\left(\chi_{k}(\tau)-\chi_{k}(0)\right)^{\top} U_{2}\left[\begin{array}{c}
\chi_{k}\left(T_{k}\right) \\
\chi_{k}(0)
\end{array}\right] \\
& +2 \dot{\chi}_{k}^{\top}(\tau)\left(\tau U_{1}+\left(T_{k}-\tau\right) U_{2}\right)\left[\begin{array}{c}
\chi_{k}\left(T_{k}\right) \\
\chi_{k}(0)
\end{array}\right] \\
& +T_{k}^{2} \dot{\chi}_{k}(\tau)^{\top} Z \dot{\chi}_{k}(\tau)-T_{k} \int_{0}^{T_{k}} \dot{\chi}_{k}(s)^{\top} Z \dot{\chi}_{k}(s) \mathrm{d} s .
\end{aligned}
$$

The previous expression can be rewritten as

$$
\begin{aligned}
& T_{k} \dot{\mathcal{V}}\left(\chi_{k}, \tau\right)=\xi_{k}^{\top}(\tau)\left[F_{00}+\tau F_{1}+\left(T_{k}-\tau\right) F_{2}\right. \\
& \left.+T_{k}^{2} M_{0}^{\top} Z M_{0}\right] \xi_{k}(\tau) \\
& -T_{k} \int_{0}^{T_{k}} \dot{\chi}_{k}(s)^{\top} Z \dot{\chi}_{k}(s) \mathrm{d} s
\end{aligned}
$$

where the matrices $F_{00}, F_{1}, F_{2}$ are defined in Theorem 4.1. Splitting then the integral into two parts and applying Jensen's inequality yields

$$
\begin{aligned}
T_{k} \dot{\mathcal{V}}\left(\chi_{k}, \tau\right) & \leq \xi_{k}^{\top}(\tau)\left[F_{00}+\tau F_{1}+\left(T_{k}-\tau\right) F_{2}\right. \\
+ & T_{k}^{2} M_{0}^{\top} Z M_{0} \\
& \left.-\left[\begin{array}{c}
-M_{12} \\
M_{13}
\end{array}\right]\left[\begin{array}{cc}
\frac{T_{k}}{T_{k}-\tau} Z & 0 \\
0 & \frac{T_{k}}{\tau} Z
\end{array}\right]\left[\begin{array}{c}
-M_{12} \\
M_{13}
\end{array}\right]\right] \xi_{k}(\tau) .
\end{aligned}
$$


where the matrices $F_{00}, F_{1}, F_{2}$ are defined in Theorem 4.1. By applying then Lemma 3.1 with $\alpha=1-\tau / T_{k}$, we then obtain

$$
\bar{M}^{\top}\left[\begin{array}{cc}
\frac{T_{k}}{T_{k}-\tau} Z & 0 \\
0 & \frac{T_{k}}{\tau} Z
\end{array}\right] \bar{M} \succeq \bar{M}^{\top}\left[\begin{array}{c}
I \\
-I
\end{array}\right] Z\left[\begin{array}{c}
I \\
-I
\end{array}\right]^{\top} \bar{M}=F_{02}
$$

where $\bar{M}=\left[\begin{array}{ll}-M_{12}^{\top} & M_{13}^{\top}\end{array}\right]^{\top}$. Using the above bound in (21) yields

$$
\begin{gathered}
T_{k} \dot{\mathcal{V}}\left(\chi_{k}, \tau\right) \leq \xi_{k}^{\top}(\tau)\left[F_{00}-F_{02}+\tau F_{1}+\left(T_{k}-\tau\right) F_{2}\right. \\
\left.+T_{k}^{2} M_{0}^{\top} Z M_{0}\right] \xi_{k}(\tau)
\end{gathered}
$$

By finally combining (17), (18) and (22) together, we obtain that

$$
\begin{aligned}
\dot{\mathcal{W}}_{k} \leq \frac{1}{T_{k}} \xi_{k}(\tau)^{\top} & {\left[F_{0}\left(T_{k}\right)+\tau F_{1}+\left(T_{k}-\tau\right) F_{2}\right.} \\
& \left.\left.+T_{k}^{2} M_{0}^{\top} Z M_{0}\right)\right] \xi_{k}(\tau) .
\end{aligned}
$$

A Schur complement yields that the (3) is asymptotically stable if the LMI

$$
\left[\begin{array}{cc}
F_{0}\left(T_{k}\right)+\tau F_{1}+\left(T_{k}-\tau\right) F_{2} & T_{k} M_{0}^{\top} Z \\
* & -Z
\end{array}\right] \prec 0
$$

is negative definite for all $\tau \in\left[0, T_{k}\right]$ and all $T_{k} \in$ $\left[T_{\min }, T_{\max }\right]$. Since the above LMI is affine in $\tau$ (hence convex), to check its negative definiteness over the entire interval $\left[0, T_{k}\right]$, it is necessary and sufficient to check it at the vertices of the set, that is only over the finite set $\tau \in\left\{0, T_{k}\right\}$, which yields the two conditions $\Phi_{1}\left(T_{k}\right) \prec 0$ and $\Phi_{2}\left(T_{k}\right) \prec 0$. The end of the proof is obtained by noting that the LMIs $\Phi_{1}\left(T_{k}\right) \prec 0$ and $\Phi_{2}\left(T_{k}\right) \prec 0$ are also affine in $T_{k}$ and the same procedure applies with the set $\left[T_{\min }, T_{\max }\right]$, finally yielding the conditions (14). The proof is complete.

\section{REFERENCES}

[1] T. Chen and B.A. Francis. Optimal sampled-data control systems. Springer-Verlag, Berlin, Germany, 1995.

[2] R. H. Middleton and G. C. Goodwin. Digital Control and Estimation - A Unified Approach. Prentice-Hall, 1990.

[3] J.P. Hespanha, P. Naghshtabrizi, and Y. Xu. A survey of recent results in networked control systems. Proceedings of the IEEE, 95(1):138162, 2007.

[4] S. Zampieri. A survey of recent results in Networked Control Systems. In Proc. of the $17^{\text {th }}$ IFAC World Congress, pages 2886 - 2894, Seoul, Korea, 2008.

[5] Y.S. Suh. Stability and stabilization of nonuniform sampling systems. Automatica, 44(12):3222-3226, 2008.

[6] Y. Oishi and H. Fujioka. Stability and stabilization of aperiodic sampled-data control systems: An approach using robust linear matrix inequalities. In Joint $48^{\text {th }}$ IEEE Conference on Decision and Control and $28^{\text {th }}$ Chinese Control Conference, pages 8142 - 8147, 2009.

[7] L. Hetel, J. Daafouz, and C. Iung. Stabilization of arbitrary switched linear systems with unknown time-varying delays. IEEE Trans. on Automatic Control, 51(10):1668-1674, Oct. 2006.

[8] M.B.G. Cloosterman, N. van de Wouw, W.P.M.H. Heemels, and H. Nijmeijer. Stability of networked control systems with uncertain time-varying delays. IEEE Trans. on Automatic Control, 54(7):1575$1580,2009$.

[9] E. Fridman, A. Seuret, and J.-P. Richard. Robust sampled-data stabilization of linear systems: An input delay approach. Automatica, 40(8):1141-1446, 2004

[10] E. Fridman. A refined input delay approach to sampled-data control. Automatica, 46(2):421-427, 2010.

[11] K. Liu and E. Fridman. Discontinuous Lyapunov functionals for linear systems with sawtooth delays. In Proc. of the $8^{\text {th }}$ IFAC Workshop on Time-Delay Systems, September 2009.
[12] A. Seuret. Stability analysis for sampled-data systems with a timevarying period. In Proc. of the $48^{\text {th }}$ IEEE Conference on Decision and Control, pages 8130 - 8135, 2009.

[13] L. Mirkin. Some remarks on the use of time-varying delay to model sample-and-hold circuits. IEEE Trans. on Automatic Control, 52(6):1009-1112, 2007.

[14] H. Fujioka. Stability analysis of systems with aperiodic sample-andhold devices. Automatica, 45(3):771-775, 2009.

[15] C.-Y. Kao and H. Fujioka. On stability of systems with aperiodic sampling devices. IEEE Transactions on Automatic Control, 58(3):20852090, 2013.

[16] C.-Y. Kao and D.-R. Wu. On robust stability of aperiodic sampleddata systems - an integral quadratic constraint approach. In American Control Conference, pages 4871-4876, Portland, USA, 2014.

[17] Y. Ariba, C. Briat, and K. H. Johansson. Simple conditions for $L_{2}$ stability and stabilization of networked control systems. In 18th IFAC World Congress, pages 96-101, Milano, Italy, 2011.

[18] P. Naghshtabrizi, J.P. Hespanha, and A.R. Teel. Exponential stability of impulsive systems with application to uncertain sampled-data systems. Systems \& Control Letters, 57(5):378-385, 2008.

[19] C. Briat. Convex conditions for robust stability analysis and stabilization of linear aperiodic impulsive and sampled-data systems under dwell-time constraints. Automatica, 49:3449-3457, 2013.

[20] A. Seuret. A novel stability analysis of linear systems under asynchronous samplings. Automatica, 48:177-182, 2012.

[21] C. Briat and A. Seuret. A looped-functional approach for robust stability analysis of linear impulsive systems. Systems \& Control Letters, 61:980-988, 2012.

[22] C. Briat and A. Seuret. Convex dwell-time characterizations for uncertain linear impulsive systems. IEEE Transactions on Automatic Control, 57(12):3241-3246, 2012.

[23] A. Seuret. Stability analysis of networked control systems with asynchronous sampling and input delay. In American Control Conference (ACC), 2011, pages 533-538. IEEE, 2011.

[24] K. Liu and E Fridman. Wirtinger's inequality and Lyapunov-based sampled-data stabilization. Automatica, 48(1):102-108, 2012.

[25] A. Seuret and C. Briat. Stability analysis of uncertain sampled-data systems with incremental delay using looped-functionals. Submitted to Automatica, 2014.

[26] K. Liu and E. Fridman. Wirtinger's inequality and Lyapunov-based sampled-data stabilization. Automatica, 48(1):102-108, 2012.

[27] P. Naghshtabrizi, J.P. Hespanha, and A.R. Teel. Stability of delay impulsive systems with application to networked control systems. Transactions of the Institute of Measurement and Control, 32(5):511528, 2010.

[28] P.G. Park, J.W. Ko, and C. Jeong. Reciprocally convex approach to stability of systems with time-varying delays. Automatica, 47(1):235238, 2011.

[29] A. Seuret and F. Gouaisbaut. Integral inequality for time-varying delay systems. In Control Conference (ECC), 2013 European, pages 33603365. IEEE, 2013.

[30] S. Boyd, L. El-Ghaoui, E. Feron, and V. Balakrishnan. Linear Matrix Inequalities in Systems and Control Theory. PA, SIAM, Philadelphia, 1994.

[31] Y. Yamamoto. New approach to sampled-data control systems - a function space method. In 29th IEEE Conference on Decision and Control, Honolulu, Hawai, pages 1882-1887, 1990.

[32] W. Zhang, M.S. Branicky, and S.M. Phillips. Stability of Networked Control Systems. IEEE Control Systems Magazine, 21(1):84-99, 2001.

[33] K. Liu and E. Fridman. Networked-based stabilization via discontinuous lyapunov functionals. Int. Journal of Robust and Nonlinear Control, 22:420-436, 2012. 